(2) Open Access Full Text Article

\title{
Intrascleral Intraocular Lens Fixation Preserving the Lens Capsule in Cases of Cataract with Insufficient Zonular Support
}

\author{
Mutsuko Kato (D) \\ Michie Namba \\ Sachika Shimoyama \\ Mayumi Inoue \\ Chihiro Ouchi \\ Takehiro Shimizu
}

Department of Ophthalmology, Japan Red Cross Okayama Hospital, Okayama, Japan
Correspondence: Mutsuko Kato Department of Ophthalmology, Japan Red Cross Okayama Hospital, 2-I-I Aoe, Kita-ward, Okayama, 700-8607, Japan Tel +8I 8622288 II

Fax +8I 86222884 I

Email mutsukato@okayama-med.jrc.or.jp
Purpose: To report our modified simple technique for optic capture and the clinical results of intrascleral IOL fixation preserving the lens capsule, without vitrectomy, in cases of cataract with insufficient zonular support to stabilize the intraocular lens (IOL).

Patients and Methods: In 37 eyes of 25 patients with phacodonesis and two or more risk factors for progressive zonular insufficiency, we inserted a CTR to support the capsule and zonules during cataract surgery and IOL fixation; an optic was inserted into the lens capsule, and a haptic was fixed in the scleral tunnel without vitrectomy. In all cases, anterior or total vitrectomy was not needed.

Results: The postoperative mean ( \pm standard deviation) tilt and decentration of the implanted IOL did not change from 6 to 12 months $\left(6.77 \pm 3.15^{\circ}\right.$ to $6.33 \pm 3.38^{\circ}$ and $0.60 \pm 0.30$ to $0.61 \pm 0.35 \mathrm{~mm}$, respectively). We encountered no late IOL dislocation and no retinal complications, including retinal breaks or cystoid macular oedema, postoperatively (follow-up $=21.1 \pm 5.2$ months).

Conclusion: Our modified techniques preclude the need for vitrectomy. If the lens capsule can be preserved using a CTR, our modified technique can be used to stabilize IOL.

Keywords: insufficient zonular support, phacodonesis, lens capsule, capsular tension ring, intrascleral IOL fixation

\section{Introduction}

In recent years, more frequent late intraocular lens (IOL)-capsular bag complex dislocation has become a major problem. ${ }^{1-4}$ This is partly attributable to the increased number of patients considered suitable for IOL implantation, even without sufficient zonular support, due to recent developments in cataract surgery ${ }^{5}$ and the use of devices including capsular tension rings $(\mathrm{CTRs})^{6,7}$ and/or capsule expanders. ${ }^{8}$ Also, earlier surgical intervention and increased life expectancy are extending the postoperative period. In cases lacking sufficient zonular support, simple CTR insertion followed by subsequent IOL implantation in a bag may be inappropriate, since many reports have described dislocation of IOL-capsular bag-CTR complexes. ${ }^{9,10}$

Suture fixation of the lens capsule using a Cionni CTR, ${ }^{11}$ capsular anchor, ${ }^{12}$ or polypropylene capsular hooks ${ }^{13}$ is helpful when zonular support is lacking, but surgery becomes difficult and complex. Posterior optic capture can be used for IOL fixation in such cases, ${ }^{14}$ with subsequent fibrosis of the anterior capsule ensuring capture stability. However, IOL stability may be compromised if the zonular support becomes insufficient during a long postoperative course. Over the past two decades, various intrascleral IOL fixation techniques have been developed and 
modified to treat such cases. ${ }^{15-20}$ These techniques are useful but increase the operation time ${ }^{21}$ and difficulty, especially for cataract surgeons, because both capsulectomy and vitrectomy are required, unlike the usual in-thebag IOL implantation or posterior optic capture of the IOL. Also, tilting and decentration of the implanted IOL often induce refractive error and high-order aberrations, which affect visual function, ${ }^{22,23}$ and the techniques carry a risk of retinal complications including retinal break, retinal detachment and cystoid macular oedema. ${ }^{15-18}$ Therefore, the ever-increasing need for cataract surgery in patients with insufficient zonular support has become a major problem. We thus developed a modified capsulepreserving intrascleral IOL fixation technique using a CTR to stabilize the IOL. Herein, we report on the utility and safety of our modified technique, and the outcomes.

\section{Patients and Methods}

\section{Patients}

This retrospective interventional case series reviewed data from the Japan Red Cross Okayama Hospital.

We studied 37 consecutive eyes ( 25 patients) treated via our modified capsule-preserving intrascleral IOL fixation technique using a CTR in the Department of Ophthalmology, Japan Red Cross Okayama Hospital, between May 2019 and August 2020; all were followed-up for at least 12 months. All of the patients had phacodonesis and exhibited at least two risk factors for progressive zonular insufficiency among the following: age $<80$ years, advanced nuclear cataracts (Emery grade $>4$ ), history of glaucoma attack, narrow angle and/or poor mydriasis, diabetes mellitus, pseudoexfoliation, trauma, high myopia, iatrogenic surgical zonular damage, and atopic dermatitis (Table 1).

\section{Surgical Technique}

A CTR (CTR130A0; Hoya, Tokyo, Japan) was inserted into the lens capsule after completion of continuous curvilinear capsulorhexis (CCC) (Figure 1A). After phacoemulsification and aspiration (PEA), infusion and aspiration (I/A) were completed with the aid of the CTR. An IOL (NX70S; Santen, Osaka, Japan) was implanted in the out-of-the-bag position (Figure 1B) (Figure 2-1). A T-shaped, 2-mm long scleral tunnel for fixation of the IOL haptic was created at the 1-o'clock meridian (Figure 1C), or at both the 1- and 7-o'clock meridians if the capsular support was very weak and/or the patient was relatively young. Angle sclerotomy was performed, $2 \mathrm{~mm}$ away from the limbus, creating
Table I Patient Characteristics

\begin{tabular}{|c|c|c|}
\hline Characteristics & \multicolumn{2}{|c|}{ Values } \\
\hline Number of eyes (patients) & \multicolumn{2}{|c|}{$37(25)$} \\
\hline Age, $y$, range $($ mean $\pm S D)$ & 53-94 & $(78.3 \pm 12.1)$ \\
\hline Males/ females & & $12 / 13$ \\
\hline $\begin{array}{l}\text { Follow-up period after surgery, } m \text {, range (mean } \pm \\
\text { SD) }\end{array}$ & $13-28$ & $(21.1 \pm 5.2)$ \\
\hline Time of recognition of phacodonesis, eyes (\%) & & \\
\hline Preoperative & & $16(43.2)$ \\
\hline Intraoperative & & $21(56.8)$ \\
\hline $\begin{array}{l}\text { Risk factors for progressive zonular insufficiency, } \\
\text { eyes (\%) }\end{array}$ & & \\
\hline Age $<80$ y & & $18(48.6)$ \\
\hline Advanced nuclear cataract (Emery grade $\geq 4$ ) & & $18(48.6)$ \\
\hline Narrow angle and/or poor mydriasis & & $16(43.2)$ \\
\hline Diabetes mellitus & & $13(35.1)$ \\
\hline History of glaucoma attack & & $8(21.6)$ \\
\hline Pseudoexfoliation & & $7(18.9)$ \\
\hline Trauma & & $4(10.8)$ \\
\hline High myopia & & $3(8.1)$ \\
\hline latrogenic surgical zonular damage & & $3(8.1)$ \\
\hline Atopic dermatitis & & $2(5.4)$ \\
\hline Number of risk factors/ eye, range (mean) & $2-4$ & (2.49) \\
\hline
\end{tabular}

Abbreviations: $y$, years; SD, standard deviation; $m$, months.

a T-shaped tunnel using a 26-gauge (26-G) needle (Figure 1D). Next, the trailing haptic of the implanted IOL was pulled out through the angle sclerotomy site at the 1-o'clock meridian using 25-G forceps (Figure 1E), and the haptic tip was then inserted into the scleral tunnel (Figure 1F). The T-shaped scleral incision was sutured with 7-0 vicryl; we included a haptic (arrow) to prevent intraocular fluid leakage and the IOL from shifting (Figure 1G). Similar procedures were performed at the 7-o'clock meridian if required. The optic of the implanted IOL was gently inserted in an in-the-bag position ("optic capture") (Figure 1H) (Figure 2-2). Appropriate CCC is important to ensure optic capture and thus stable fixation of the IOL optic (and the remaining lens capsule-CTR complex) (Figure 2-4, arrow). Aggressive manoeuvres during IOL optic capture via a small CCC may compromise dialysis if the zonules are weak. After intrascleral fixation of the upper haptic (Figure 2-3, arrow head) or both haptics of the implanted IOL, the IOL-capsular bag-CTR complex was stable (Figure 2-2 and 2-4). See Supplementary Video for the surgical technique.

\section{Examination}

At all visits, all patients underwent standard ophthalmic examinations. The corrected distant visual acuities 

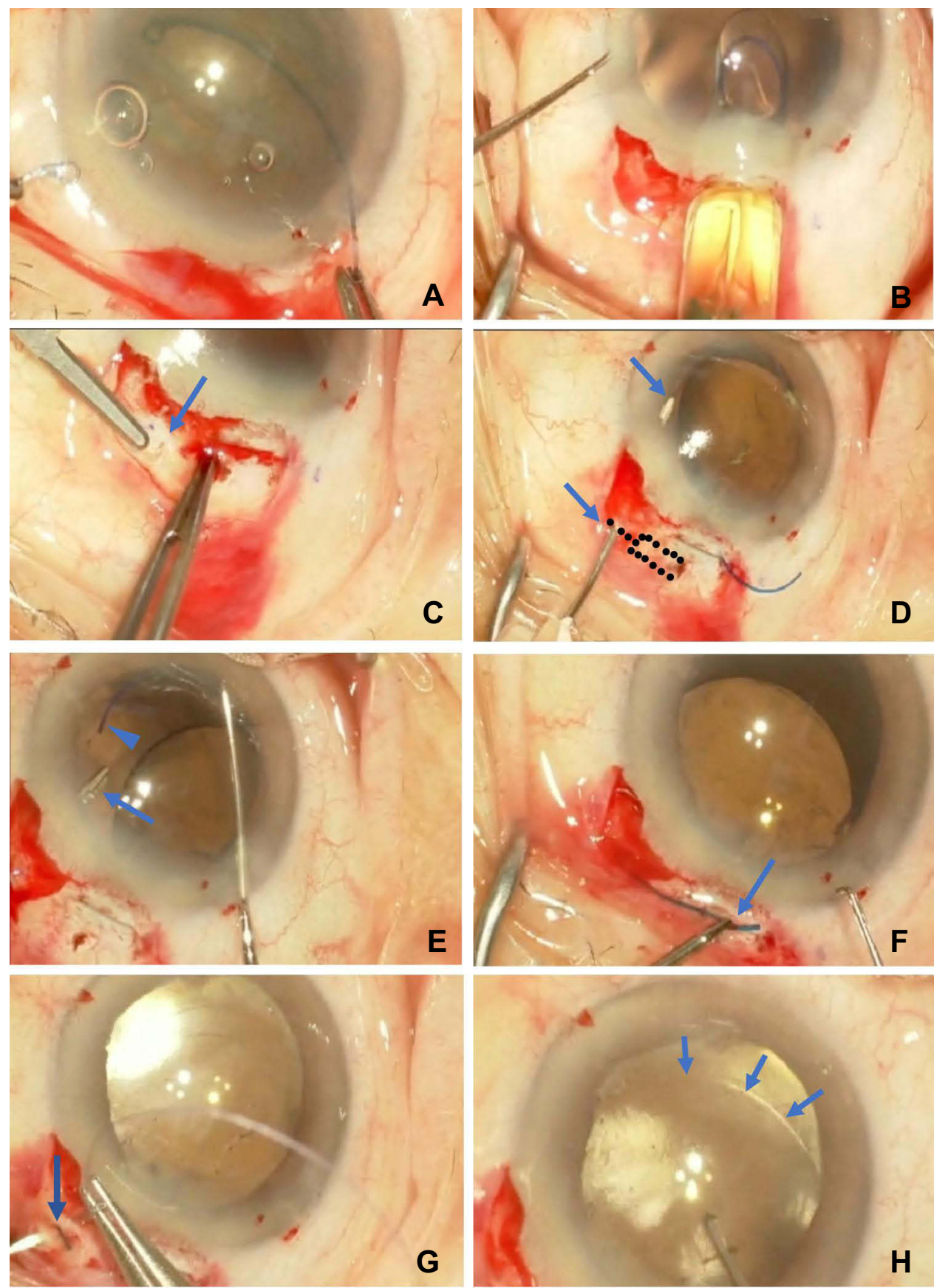

Figure I Procedure for intrascleral IOL fixation preserving the lens capsule. (A) For each case with phacodonesis, a CTR was inserted into the capsule after completion of appropriately sized CCC. PEA and I/A were completed with the aid of the CTR. (B) An IOL was implanted in the out-of-the-bag position using an injector. (C) A T-shaped 2-mm long scleral tunnel for fixation of an IOL haptic was created $2.0 \mathrm{~mm}$ from the limbus at the I-o'clock Meridian (or at both the I- and 7-o'clock meridians when the capsule support was very weak and/or the patient was relatively young). (D) Angle sclerotomy creating a T-shaped tunnel (represented by dots) was performed using a 26-G needle (arrows) parallel to the iris and above the anterior capsule. (E) The upper haptics of the IOL (arrow head) were dialled in and externalized through the sclerotomy site using 25-G forceps (arrow). (F) The tip of an IOL haptic (arrow) was inserted into the scleral tunnel ("intrascleral haptic fixation"). (G) The scleral incision was closed with a 7-0 vicryl suture; we included a haptic (arrow) to prevent intraocular fluid leakage and the IOL from shifting. $(\mathbf{H})$ The IOL optic was gently inserted into the capsular bag ("optic capture"). The arrows indicate the CCC margin.

Abbreviations: IOL, intraocular lens; CTR, capsular tension ring; CCC, continuous curvilinear capsulorhexis; PEA, phacoemulsification and aspiration; I/A, infusion and aspiration; G, gauge. 

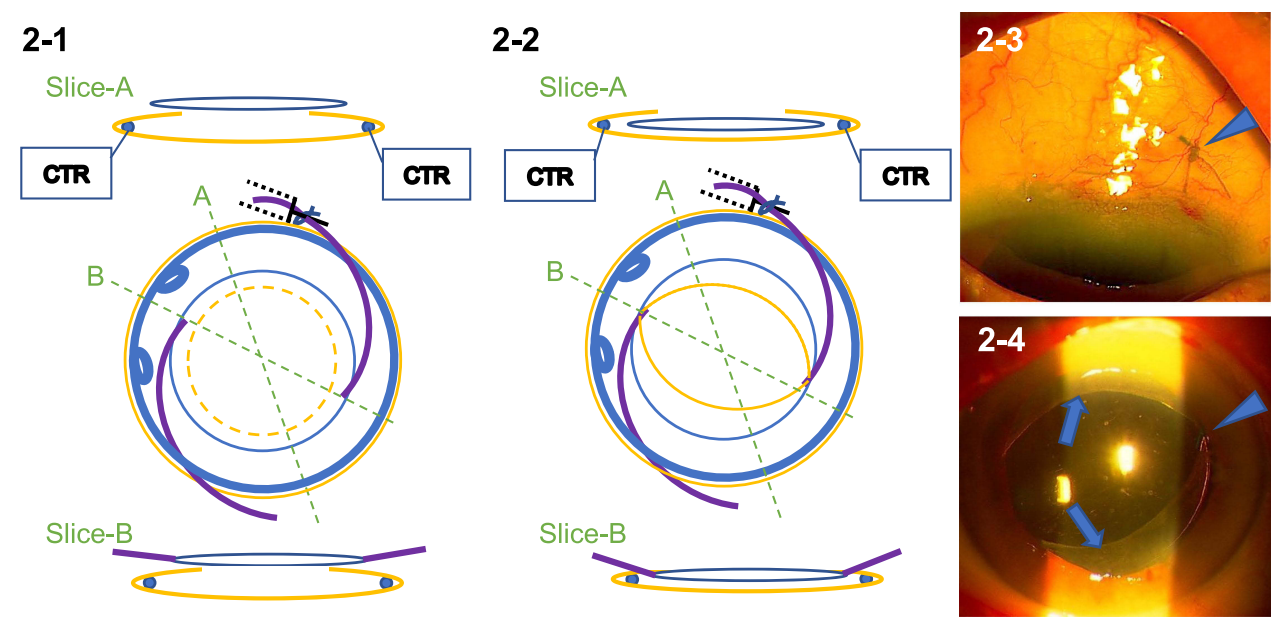

Figure 2 Schema of the intrascleral IOL fixation method preserving the lens capsule. (2-I) First, an IOL was implanted in an out-of-the-bag position. Then, the tip of the upper IOL haptic was fixed in the scleral T-shaped tunnel (the lower haptic was placed on the bag or fixed in the scleral tunnel, as was the upper haptic). (2-2) Second, the IOL optic was gently inserted into the capsular bag ("optic capture"). The IOL supports the capsule containing the CTR. Simultaneously, the spared capsule and vitreous body support the optic (to prevent IOL tilting). Slice "A" shows the optics of the implanted in-the-bag IOL and the positions of the spared capsule and CTR. Slice "B" shows that the haptics support the capsule with the CTR and that the optic is supported by the face of the spared capsule and vitreous, preventing tilting. (2-3) The arrowhead indicates the tip of the upper IOL haptic and 7-0 vicryl suture. (2-4) The arrows indicate the CCC margin, and the arrowhead indicates the loop attachment. Abbreviations: IOL, intraocular lens; CTR, capsular tension ring; CCC, continuous curvilinear capsulorhexis.

(CDVAs) (at $5 \mathrm{~m}$ ) before and after surgery were obtained using a Landolt chart. Corneal endothelial cell densities (ECDs) were measured employing the Cell Check CC7000 device (Konan Medical Inc., Nishinomiya, Japan) before and at 1 month after surgery, as were the refractive errors at 6 months after surgery, the postoperative IOL tilt and decentration at 6 and 12 months (m) after surgery (via anterior segment ocular coherence tomography; AS-OCT) (CASIA 2 TM, Tomey Corporation, Nagoya, Japan). Any postoperative complications were recorded.

\section{Statistical Analysis}

The visual acuity was the logarithm of the minimum angle of resolution. Postoperative changes in visual acuity and ECD were assessed relative to the preoperative values using the Wilcoxon signed-rank test after normalities were checked. All values are presented as means \pm standard deviations, and $P$-values of less than 0.05 were considered to denote statistical significance. All statistical analyses were performed with the aid of SPSS software for Windows (version 25.0; SPSS, Inc., Chicago, IL, USA).

\section{Results}

All cases had phacodonesis and at least two risk factors for progressive zonular weakness, as described in Table 1. Phacodonesis was diagnosed preoperatively in 16 eyes $(43.2 \%)$ and intraoperatively in 21 eyes (56.8\%). Risk factors included an age less than 80 years in 18 eyes $(48.6 \%)$, advanced nuclear cataract (Emery grade $\geq 4$ ) in 18 eyes $(48.6 \%)$, narrow angle and/or poor mydriasis in 16 eyes $(43.2 \%)$, diabetes mellitus in 13 eyes $(35.1 \%)$, post glaucoma attack in 8 eyes $(21.6 \%)$, pseudoexfoliation in 7 eyes $(18.9 \%)$, trauma in 4 eyes $(10.8 \%)$, high myopia in 3 eyes $(8.1 \%)$, iatrogenic surgical zonular damage in 3 eyes $(8.1 \%)$ and atopic dermatitis in 2 eyes $(5.4 \%)$. The number of risk factors for zonular insufficiency per eye ranged from $2-4$ (mean $=2.49)$. Using our modified technique, in 26 of the 37 eyes $(70.3 \%)$ only the upper haptic was fixed with capsule-preserving fixation (single haptic fixation). In the remaining 11 eyes (29.7\%), because of the extreme weak capsular supports and/or relatively young age of the patients, both the upper and lower haptics were fixed to the scleral tunnel (dual haptic fixation). There was no significant difference in patient characteristics between the single and dual haptic fixation groups (Table S1).

Table 2 shows the postoperative data. The mean preoperative CDVA was $0.75 \pm 0.82 \log$ MAR, which improved significantly to $0.20 \pm 0.40 \log \mathrm{MAR}$ at the last visit $(P<0.01)$. In 32 eyes $(86.5 \%)$ the CDVA improved, and in 5 eyes $(13.5 \%)$ it remained stable. The mean preoperative ECD was $2409 \pm 459$ cells/ $\mathrm{mm}^{2}$, which decreased significantly to $2191 \pm 500$ cells $/ \mathrm{mm}^{2}(P<0.01)$ The mean decrease in ECD was $10.3 \pm 11.4 \%$, and there was no difference in the degree 
Table 2 Summary of Surgical Outcomes

\begin{tabular}{|c|c|c|c|}
\hline \multirow{2}{*}{$\begin{array}{l}\text { Characteristics } \\
\text { CDVA (logMAR) (mean } \pm S D)\end{array}$} & \multirow[t]{2}{*}{ After Surgery } & \multicolumn{2}{|l|}{ Values } \\
\hline & & & \\
\hline Preoperative & & & $(0.75 \pm 0.82)$ \\
\hline Postoperative & (at last visit) & & $(0.20 \pm 0.40)^{* * *}$ \\
\hline \multicolumn{4}{|l|}{ Endothelial cell density, cells $/ \mathrm{mm}^{2}$ (mean $\pm \mathrm{SD}$ ) } \\
\hline Preoperative & & & $(2409 \pm 459)$ \\
\hline Postoperative & (at I m) & & $(2191 \pm 500)^{* * *}$ \\
\hline Percent change in ECD, \%, range (mean $\pm \mathrm{SD}$ ) & & $0-43.8$ & $(10.3 \pm 11.4)$ \\
\hline Prediction error of the target refraction, dioptre, range (mean $\pm S D$ ) & (at $6 \mathrm{~m}$ ) & $+1.47 \sim-2.08$ & $(-0.64 \pm 0.97)$ \\
\hline Percent of myopic deviation, eyes (\%) & & & $29(78.4 \%)$ \\
\hline \multirow[t]{2}{*}{ Tilt angle of $\mathrm{IOL}$, degrees, range (mean $\pm \mathrm{SD}$ ) } & (at $6 \mathrm{~m})$ & $1.4-13.3$ & $(6.77 \pm 3.15)$ \\
\hline & (at $12 \mathrm{~m}$ ) & $0.7-14.0$ & $(6.33 \pm 3.38)$ \\
\hline \multirow[t]{2}{*}{ Decentration of IOL, $\mathrm{mm}$, range (mean $\pm \mathrm{SD}$ ) } & (at $6 \mathrm{~m}$ ) & $0.11-1.42$ & $(0.60 \pm 0.30)$ \\
\hline & (at $12 \mathrm{~m}$ ) & $0.11-1.37$ & $(0.6 I \pm 0.35)$ \\
\hline \multicolumn{4}{|l|}{ Postoperative complications, eyes (\%) } \\
\hline Smooth vitreous haemorrhage & & & $2(5.4)$ \\
\hline Rupture of the lens capsule & & & I (2.7) \\
\hline Transient ocular hypertension & & & I (2.7) \\
\hline
\end{tabular}

Notes: $* * *$ Wilcoxon signed-rank test, $\mathrm{P}<0.001$

Abbreviations: CDVA, corrected distance visual acuity; logMAR, logarithm of minimum angle of resolution; SD, standard deviation; m, months; ECD, endothelial cell density; IOL, intraocular lens.

of change between the single and dual fixation groups. The mean refractive difference from the predicted value was $-0.64 \pm 0.97 \mathrm{D}$ (range: $+1.47 \sim-2.08$ ). Most of the eyes (29 eyes, 78.4\%) showed a tendency toward myopic deviation. In the dual fixation group, the mean prediction error $(-1.22 \pm 0.76 \mathrm{D})$ was significantly larger than in the single fixation group $(-0.40 \pm 0.94 \mathrm{D})$ $(P=0.018)$ (Table S2). The postoperative mean tilt and decentration of the implanted IOL did not change from 6 to 12 months $\left(6.77 \pm 3.15^{\circ}\right.$ to $6.33 \pm 3.38^{\circ}, 0.60 \pm$ $0.30 \mathrm{~mm}$ to $0.61 \pm 0.35 \mathrm{~mm}$, respectively), and there was no significant difference between the single and dual fixation groups.

Postoperative smooth vitreous haemorrhage developed in $2(5.4 \%)$ of the 37 eyes but resolved spontaneously within 2 weeks. Postoperative transient ocular hypertension was evident in one eye, and rupture of the lens capsule was seen in another. Although rupture of the lens capsule was seen during IOL optic capture via a small CCC in one eye, the IOL was fixed using our modified technique because of no vitreous prolapse. We encountered no postoperative retinal complications (including retinal breaks and cystoid macular oedema), hypotony, severe refractive error (necessitating IOL replacement), large tilt requiring haptic adjustment, or late in-the-bag IOL dislocation. Subsequently, posterior capsule opacification was seen in 2 eyes (5.4\%) and both eyes underwent posterior capsulotomy. Tilting and decentration of both did not change within the observation period of this study.

\section{Discussion}

The cumulative risk of late IOL-capsular bag complex dislocation is $0.1 \%$ at 10 years and $1.7 \%$ at 25 years after cataract surgery; thus, the risk increases over time. ${ }^{2,3}$ Some authors found that the increase was primarily attributable to the longer duration of pseudophakia and recent cataract surgery. Dabrowska et al reported that the cumulative risks at $5,10,15$, and 20 years after cataract surgery were $0.09 \%, 0.55 \%, 1.00 \%$, and $1.00 \%$, respectively, and were significantly higher $(P<0.001)$ for eyes that had undergone cataract surgery between 2002 and 2012 than for eyes operated on between 1992 and 2001 $(0.89 \%$ vs $0.39 \%$ at 10 years postoperatively) $(P<$ $0.001){ }^{3}$ Some authors reported dislocation of the IOLcapsular bag complex even when CTRs were used., ${ }^{9,10}$ The aetiologies of late IOL-capsular bag complex dislocation include aging, high myopia, uveitis, trauma, retinitis pigmentosa, diabetes mellitus, atopic dermatitis, connective tissue disorders, and previous vitreoretinal surgery or an acute attack of angle-closure glaucoma. ${ }^{2}$ Thus, we applied our modified technique in cases of phacodonesis with at least two risk factors of progressive zonular weakness, as described in Table 1, to stabilize IOL. Also, in this study, we regarded the longer postoperative period (age $<$ 
80 years) as another potential risk factor for late onset of IOL dislocation.

The first advantage of our method is the non-necessity for vitrectomy and preservation of the lens capsule; this reduces the risk of retinal complications including retinal breaks, retinal detachment, and cystoid macular oedema. ${ }^{15-18}$ Only posterior optic capture can be used easily for IOL fixation in cases lacking sufficient capsular support, ${ }^{14}$ but there remains a risk of postoperative progression of zonular weakening and resultant IOL tilting and decentration long after the surgery. Suture fixation of the lens capsule using a Cionni CTR, ${ }^{11}$ capsular anchor, ${ }^{12}$ or polypropylene capsular hooks ${ }^{13}$ in cases of insufficient zonular support are more complicated methods and have some severe postoperative complications, including pseudophacodonesis, decentration, increased intraocular pressure, persistent iritis, broken suture, and retinal detachment. ${ }^{11-13,24}$ Thus, we combined optic capture (in-the-bag fixation of the IOL) with the use of a CTR and intrascleral haptic fixation, by preserving the lens capsule without vitrectomy. Cataract surgeons can easily and rapidly apply our technique, even in cases lacking sufficient zonular support.

Postoperative visual function deterioration is significantly associated with IOL malpositioning and refractive errors, as well as with high-order aberrations caused by IOL tilting and decentration. ${ }^{22,23,25}$ Many reports have found that conventional intrascleral IOL fixation ${ }^{23,25,26}$ combined with capsulectomy and vitrectomy can result in large refractive errors and high-order aberrations. The postoperative refractive prediction error in this study was $-0.68 \pm 0.95 \mathrm{D}$ (myopia in $78.4 \%$ of cases), and was larger in the dual haptic fixation group. During the transscleral procedure, in which the haptics were sutured $1.5 \mathrm{~mm}$ from the corneal limbus, Ahn et al reported a $-1.0 \mathrm{D}$ myopic shift. ${ }^{27}$ Yamane et al made scleral tunnels $2 \mathrm{~mm}$ from the limbus, reported the needle insertion angle was also important and the mean prediction error was $-0.21 \pm$ 0.99 D. $^{18}$ Jujo et al reported a mean prediction error of $-0.03 \pm 0.93 \mathrm{D}$ when using their $27-\mathrm{G}$ trocar-assisted sutureless IOL fixation method. ${ }^{28}$ Torii et al compared clinical outcomes between intracapsular implantation and intrascleral fixation of the same IOL; the mean error was $0.22 \pm 0.17$ and $0.86 \pm 0.59 \mathrm{D}$, and the postoperative anterior chamber depth was $4.65 \pm 0.23$ and $4.98 \pm$ $0.61 \mathrm{~mm}$, respectively. ${ }^{26}$ With our method, the IOL haptics located out-of-the-bag and fixed in the scleral tunnels might pull up the IOL optic located in-the-bag. Postoperative refraction tended toward myopia, especially in the dual haptic fixation group. Because of the small number of cases and different indications between the single and dual fixation groups, it may be necessary to validate our method in the future.

Regarding tilting and decentration, Matsumura et $\mathrm{al}^{29}$ used AS-OCT to assess the maximum IOL tilt angle and decentration relative to the corneal topographic axis; the tilt was $8.13 \pm 5.15^{\circ}$ after intrascleral fixation. Torii et $\mathrm{al}^{26}$ reported a tilt of $8.8 \pm 3.9^{\circ}$ and decentration of $0.52 \pm$ $0.35 \mathrm{~mm}$ in cases undergoing intrascleral fixation, and respective values of $5.21 \pm 1.47^{\circ}$ and $0.22 \pm 0.13 \mathrm{~mm}$ in those treated via common in-the-bag IOL implantation. In present study, the postoperative mean tilt angle $(6.77 \pm$ $\left.3.15^{\circ}\right)$ and decentration $(0.60 \pm 0.30 \mathrm{~mm})$ of the implanted IOL at 6 months did not change significantly at 12 months $\left(6.33 \pm 3.38^{\circ}\right.$ and $0.61 \pm 0.35 \mathrm{~mm}$, respectively $)$, and no late IOL-capsular bag-CTR complex dislocation was noted during the follow-up (average, 21.1 months). The second advantage of our modified method is that the IOL is supported and stabilized by the remaining capsule, CTR, and vitreous, which limit tilt and decentration. Based on this study, the persistence of the extended capsular diaphragm (the IOL-capsule-CTR complex) may prevent distortion. ${ }^{6,30}$ Some reports have suggested that a CTR may inhibit (post-cataract) lens epithelial cell migration, ${ }^{9}$ thus reducing the risk of capsulorhexis-optic contact ${ }^{6}$ and progressive IOL tilting. ${ }^{30}$ Our technique can be regarded as a modification of IOL optic capture. As reported by Gimbel and DeBroff, ${ }^{14}$ IOL optic capture can be attained via various means; all afford long-term IOL stability and centration. Our modification achieves optic capture using a preserving lens capsule combined with an CTR and intrascleral haptic fixation of the IOL, to provide mechanical support to the lens zonules and inhibit shrinkage of the remaining lens capsule (and thus zonular dehiscence). However, in the past few years, improved techniques with even less tilting and decentration have been reported, ${ }^{18,28}$ and there may be room for improvement of our technique.

Postoperative complications were less common after applying our modified capsule-preserving intrascleral IOL fixation technique than after conventional fixation with vitrectomy (data not shown); this represents the third advantage of our approach. There have been many improvements to intrascleral haptic fixation methods. In our method, the haptic is grasped with $25 \mathrm{G}$ forceps, inserted into a T-shaped scleral tunnel and then sutured. Small sclerostomies were expected to reduce the incidence 
of postoperative hypotony and ciliary body injury, ${ }^{18}$ but were instead associated with greater IOL tilt and IOL astigmatism after surgery. ${ }^{29}$ We prevented hypotony and limited IOL tilt by using $25-\mathrm{G}$ sclerostomy followed by suturing with 7-0 vicryl.

Our retrospective observational study had certain limitations, including a short follow-up period and a small number of patients. The retrospective and non-randomized nature of the work renders comparisons with optic capture alone and conventional intrascleral fixation techniques (accompanied by capsulectomy and vitrectomy) difficult. A clinical limitation is that our modified technique is not applicable if the zonular support is absent or if vitreous prolapse is evident preoperatively or intraoperatively. Also, an adequately large CCC is indispensable for optic capture of the IOL. ${ }^{14}$ Further randomized comparative clinical studies are needed.

\section{Conclusion}

Our modified technique featuring optic capture, CTR placement, and intrascleral haptic fixation is safe when used to treat cataracts via IOL implantation and valuable when capsular support is lacking; additionally, anterior vitrectomy is not required. If the capsule can be preserved with the aid of a CTR, our modified technique is worthwhile. IOL fixation is supported by the capsule, CTR, optic capture, vitreous, and intrascleral fixation of the IOL haptics. This simple, safe, and useful technique may reduce the incidence of late in-the-bag IOL dislocation in cases lacking adequate zonular support.

\section{Ethics Approval and Informed Consent}

The study protocol was approved by the Medical Ethics Committee of Japan Red Cross Okayama Hospital (approval \#2020-56). All clinical procedures adhered to the principles of the Declaration of Helsinki. Informed consent was obtained from all patients after careful explanation of the study protocol, surgical procedures, ophthalmological examinations, and possible complications.

\section{Author Contributions}

MK performed the surgery, designed the study, collected and analysed the data, and drafted the manuscript. MN, SS and MI collected data. CO assisted with the data collection, and TS assisted with the statistical analysis. All authors made substantial contributions to conception and design, acquisition of data, or analysis and interpretation of data; took part in drafting the article or revising it critically for important intellectual content; agreed to submit to the current journal; gave final approval of the version to be published; and agree to be accountable for all aspects of the work.

\section{Disclosure}

The authors report no conflicts of interest relevant to this work.

\section{References}

1. Gimbel HV, Condon GP, Kohnen T, et al. Late in-the-bag intraocular lens dislocation: incidence, prevention, and management. $J$ Cataract Refract Surg. 2005;31(11):2193-2204. doi:10.1016/j.jcrs.2005.06.053

2. Ascaso FJ, Huerva V, Grzybowski A. Epidemiology, etiology, and prevention of late IOL-capsular bag complex dislocation: review of the literature. J Ophthalmol. 2015;2015:805706. doi:10.1155/2015/ 805706

3. Dabrowska-Kloda K, Kloda T, Boudiaf S, et al. Incidence and risk factors of late in-the-bag intraocular lens dislocation: evaluation of 140 eyes between 1992 and 2012. J Cataract Refract Surg. 2015;41 (7):1376-1382. doi:10.1016/j.jcrs.2014.10.040

4. Mönestam E. Frequency of intraocular lens dislocation and pseudophacodonesis, 20 years after cataract surgery - a prospective study. Am J Ophthalmol. 2019;198:215-222. doi:10.1016/j.ajo.2018.10.020

5. Lee G-I, Lim DH, Chi SA, et al. Risk factors for intraocular lens dislocation after phacoemulsification: a nationwide population-based cohort study. Am J Ophthalmol. 2020;214:86-96. doi:10.1016/j. ajo.2020.03.012

6. Menapace R, Findl O, Georgopoulos M, et al. The capsular tension ring: designs, applications, and techniques. J Cataract Refract Surg. 2000;26(6):898-912. doi:10.1016/S0886-3350(00)00446-6

7. Hasanee K, Butler M, Ahmed IIK. Capsular tension rings and related devices: current concepts. Curr Opin Ophthalmol. 2006;17(1):31-41. doi:10.1097/01.icu.0000193069.32369.e1

8. Asano Y, Yaguchi S, Nishimura E, et al. Modified capsule expander implantation to reposition and fixate the capsular bag in eyes with subluxated cataractous lenses and phacodonesis: intermediate-term results. J Cataract Refract Surg. 2015;41(3):598-606. doi:10.1016/j. jers.2014.06.031

9. Moreno-Montañés J, Heras H, Fernández-Hortelano A. Surgical treatment of a dislocated intraocular lens-capsular bag-capsular tension ring complex. J Cataract Refract Surg. 2005;31(2):270-273. doi:10.1016/j.jcrs.2004.04.041

10. Werner L, Zaugg B, Neuhann T, et al. In-the-bag capsular tension ring and intraocular lens subluxation or dislocation: a series of 23 cases. Ophthalmol. 2012;119(2):266-271. doi:10.1016/j. ophtha.2011.08.016

11. Cionni RJ, Osher RH, Marques DMV, et al. Modified capsular tension ring for patients with congenital loss of zonular support. J Cataract Refract Surg. 2003;29(9):1668-1673. doi:10.1016/s08863350(03)00238-4

12. Ton Y, Naftali M, Gortzak RL, et al. Management of subluxated capsular bag-fixated intraocular lenses using a capsular anchor. $J$ Cataract Refract Surg. 2016;42(5):653-658. doi:10.1016/j. jcrs.2016.04.002

13. Jin H, Ou Z, Zhang Q, et al. Intrascleral fixation of implantable polypropylene capsular hook(s): a new sutureless technique to reposition dislocated intraocular lens-capsular bag complex. Retina. 2019;39(1):S44-S49. doi:10.1097/IAE.0000000000001915 
14. Gimbel HV, DeBroff BM. Intraocular lens optic capture. $J$ Cataract Refract Surg. 2004;30(1):200-206. doi:10.1016/j.jcrs.2003.11.035

15. Gabor SGB, Pavlidis MM. Sutureless intrascleral posterior chamber intraocular lens fixation. $J$ Cataract Refract Surg. 2007;33 (11):1851-1854. doi:10.1016/j.jcrs.2007.07.013

16. Ohta T, Toshida H, Murakami A. Simplified and safe method of sutureless intrascleral posterior chamber intraocular lens fixation: y-fixation technique. $J$ Cataract Refract Surg. 2014;40(1):2-7. doi:10.1016/j.jcrs.2013.11.003

17. Yamane S, Inoue M, Arakawa A, et al. Sutureless 27-gauge needle-guided intrascleral intraocular lens implantation with lamellar scleral dissection. Ophthalmol. 2014;121(1):61-66. doi:10.1016/j. ophtha.2013.08.043

18. Yamane S, Sato S, Maruyama-Inoue M, et al. Flanged intrascleral intraocular lens fixation with double-needle technique. Ophthalmol. 2017;124(8):1136-1142. doi:10.1016/j.ophtha.2017.03.036

19. Scharioth GB, Prasad S, Georgalas I, et al. Intermediate results of sutureless intrascleral posterior chamber intraocular lens fixation. $J$ Cataract Refract Surg. 2010;36(2):254-259. doi:10.1016/j.jcrs.2009.09.024

20. Agarwal A, Kumar DA, Jacob S, et al. Fibrin glue-assisted sutureless posterior chamber intraocular lens implantation in eyes with deficient posterior capsules. J Cataract Refract Surg. 2008;34(9):1433-1438. doi:10.1016/j.jcrs.2008.04.040

21. Suzuki Y, Tando T, Adachi K, et al. Modified intraocular lens intrascleral fixation technique using two vitrectomy ports as lens haptic fixation sites. Clin Ophthalmol. 2020;14:1223-1228. doi:10.2147/OPTH.S247920

22. Oshika T, Sugita G, Miyata K, et al. Influence of tilt and decentration of scleral-sutured intraocular lens on ocular higher-order wavefront aberration. British J Ophthalmol. 2007;91(2):185-188. doi:10.1136/ bjo.2006.099945

23. Aizawa N, Kunikata H, Maeda N, et al. Analysis of higher-order aberrations in eyes having undergone intraocular lens suturing. Clin Exp Ophthalmol. 2015;43(1):92-94. doi:10.1111/ceo.12366
24. Cai L, Han X, Jiang Y, et al. Three-year outcomes of cionni-modified capsular tension ring implantation in children under 8 years old with ectopia lentis. Am J Ophthalmol. 2021;224:74-83. doi:10.1016/j. ajo.2020.11.011

25. Kurimori HY, Inoue M, Hirakata A. Adjustments of haptics length for tilted intraocular lens after intrascleral fixation. Am J Ophthalmol Case Rep. 2018;10:180-184. doi:10.1016/j.ajoc.2018.02.025

26. Torii T, Tamaoki A, Kojima T, et al. Comparison of clinical outcomes between intracapsular implantation and intrascleral fixation using the same model of intraocular lens. Clin Ophthalmol. 2020;14:3965-3974. doi:10.2147/OPTH.S268126

27. Ahn JK, Yu HG, Hum Chung M, et al. Transscleral fixation of a foldable intraocular lens in aphakic vitrectomized eyes. J Cataract Refract Surg. 2003;29(12):2390-2396. doi:10.1016/ S0886-3350(03)00338-9

28. Jujo T, Kogo J, Sasaki H, et al. 27-gauge trocar-assisted sutureless intraocular lens fixation. BMC Ophthalmol. 2021;21(1):8. doi:10.1186/s12886-020-01758-6

29. Matsumura T, Takamura Y, Makita J, et al. Influence of sclerotomy size on intraocular lens tilt after intrascleral intraocular lens fixation. $J$ Cataract Refract Surg. 2019;45(10):1446-1451. doi:10.1016/j. jers.2019.06.006

30. Yang S, Jiang H, Nie $\mathrm{K}$, et al. Effect of capsular tension ring implantation on capsular stability after phacoemulsification in patients with weak zonules: a randomized controlled trial. CTR implantation in cataract patients with weak zonules. $B M C$ Ophthalmol. 2021;21(1):19. doi:10.1186/s12886-020-01772-8
Clinical Ophthalmology

\section{Publish your work in this journal}

Clinical Ophthalmology is an international, peer-reviewed journal covering all subspecialties within ophthalmology. Key topics include: Optometry; Visual science; Pharmacology and drug therapy in eye diseases; Basic Sciences; Primary and Secondary eye care; Patient Safety and Quality of Care Improvements. This journal is indexed on PubMed

\section{Dovepress}

Central and CAS, and is the official journal of The Society of Clinical Ophthalmology (SCO). The manuscript management system is completely online and includes a very quick and fair peer-review system, which is all easy to use. Visit http://www.dovepress.com/ testimonials.php to read real quotes from published authors. 